\title{
Structural and biochemical studies on Pompe disease and a "pseudodeficiency of acid $\alpha$-glucosidase"
}

\author{
Youichi Tajima $\cdot$ Fumiko Matsuzawa $\cdot$ Sei-ichi Aikawa $\cdot$ Toshika Okumiya $\cdot$ \\ Michiru Yoshimizu - Takahiro Tsukimura $\cdot$ Masahiko Ikekita \\ Seiichi Tsujino $\cdot$ Akihiko Tsuji $\cdot$ Tim Edmunds $\cdot$ Hitoshi Sakuraba
}

Received: 16 April 2007/ Accepted: 10 August 2007/Published online: 6 September 2007

(C) The Japan Society of Human Genetics and Springer 2007

\begin{abstract}
We constructed structural models of the catalytic domain and the surrounding region of human wildtype acid $\alpha$-glucosidase and the enzyme with amino acid substitutions by means of homology modeling, and examined whether the amino acid replacements caused structural and biochemical changes in the enzyme proteins. Missense mutations including p.R600C, p.S619R and p.R437C are predicted to cause apparent structural changes. Nonsense mutation of p.C103X terminates the translation of acid $\alpha$-glucosidase halfway through its biosynthesis and is deduced not to allow formation of the active site pocket. The mutant proteins resulting from these missense and nonsense mutations found in patients with Pompe disease are predictably unstable and degraded quickly in cells. The structural change caused by p.G576S is predicted to be small, and cells from a subject homozygous for this amino acid substitution exhibited 15 and $11 \%$ of the normal enzyme activity levels for an artificial substrate and
\end{abstract}

Conflict of interest: T.E. is an employee of Genzyme Corporation.

Y. Tajima $\cdot$ F. Matsuzawa $\cdot$ S. Aikawa $\cdot$ M. Yoshimizu

T. Tsukimura $\cdot$ H. Sakuraba

Department of Clinical Genetics, The Tokyo Metropolitan

Institute of Medical Science, Tokyo Metropolitan Organization

for Medical Research, Tokyo, Japan

T. Okumiya

Department of Analytical Biochemistry, School of Health

Sciences, Kumamoto University, Kumamoto, Japan

M. Yoshimizu $\cdot$ T. Tsukimura $\cdot$ M. Ikekita

Department of Applied Biological Science, Faculty of Science

and Technology, Tokyo University of Science, Noda, Japan

\section{S. Tsujino}

Department of Peripheral Nervous System Research,

National Institute of Neuroscience, NCNP, Kodaira, Japan glycogen, respectively, and corresponding amounts of the enzyme protein on Western blotting. No accumulation of glycogen was found in organs including skeletal muscle in the subject, and thus the residual enzyme activity could protect cells from glycogen storage. On the other hand, p.E689K, which is known as a neutral polymorphism, little affected the three-dimensional structure of acid $\alpha$-glucosidase. Structural study on a mutant acid $\alpha$-glucosidase in silico combined with biochemical investigation is useful for understanding the molecular pathology of Pompe disease.

Keywords Pompe disease - Glycogen $\cdot \alpha$-Glucosidase . Gene mutation · Pseudodeficiency · Protein structure

\section{Introduction}

Pompe disease (glycogen storage disease type II, acid $\alpha$ glucosidase deficiency, acid maltase deficiency; OMIM:

\footnotetext{
A. Tsuji

Department of Biological Science and Technology,

Faculty of Engineering, University of Tokushima,

Tokushima, Japan

T. Edmunds

Genzyme Corporation, Framingham, MA,

H. Sakuraba ( $\square)$

Department of Analytical Biochemistry,

Meiji Pharmaceutical University, 2-522-1 Noshio,

Kiyose, Tokyo 204-8588, Japan

e-mail: sakuraba@my-pharm.ac.jp
} 
232300) is an autosomal recessive genetic disorder resulting from a deficiency of acid $\alpha$-glucosidase (EC 3.2.1.20; Swiss-Prot Entry Name: LYAG_HUMAN, Accession number: P10253) activity (Hirschhorn and Reuser 2001). The enzyme defect causes lysosomal accumulation of glycogen. This disorder exhibits a wide clinical spectrum. The classic infantile-onset subtype is severe and characterized by systemic manifestations including cardiomegaly, hypotonia, muscular weakness and hepatomegaly. The late-onset form of the disease essentially involves the skeletal muscle and is slowly progressive. The incidence of this disease varies with the ethnic group. The frequency of the infantile-onset disease has been estimated to be $1 / 14,000$ to $1 / 137,000$, that of the late-onset disease being approximately 1/60,000 (Hirschhorn and Reuser 2001).

With the isolation and characterization of human acid $\alpha$-glucosidase cDNA (Martiniuk et al. 1986; Hoefsloot et al. 1988) and the structural gene (Hoefsloot et al. 1990; Martiniuk et al. 1991), many gene mutations causing a deficiency of acid $\alpha$-glucosidase activity, which results in Pompe disease, have been found (Hirschhorn and Reuser 2001; Hermans et al. 2004; Tsunoda et al. 1996; Tsujino et al. 2000; Kanazawa et al. 2003). Furthermore, Hwu et al. reported the results of screening for Pompe disease in Taiwan, which revealed that there are asymptomatic cases having considerably low acid $\alpha$-glucosidase activity. Gene analysis revealed that they have p.G576S and p.E689K on their alleles, the latter being a neutral polymorphism (Hwu WL, Chiang SC, Zhang XK, Keutzer J, Chien YH, Lee NC. Neonatal screening for Pompe disease: Result from the Taiwan screening program. The tenth International Congress of Inborn Errors of Metabolism, 13 September 2006, Makuhari, Japan). As enzyme replacement therapy with recombinant human acid $\alpha$-glucosidase has been introduced (Van den Hout et al. 2004; Klinge et al. 2005; Kishnani et al. 2006), it is very important to clarify the molecular pathology of Pompe disease at the protein level. Until now, however, it has been unknown how amino acid substitutions affect the structure of acid $\alpha$-glucosidase.

Human acid $\alpha$-glucosidase cDNA encodes a peptide composed of 952 amino acid residues, and its sequence contains seven potential $N$-glycosylation sites. Previous studies revealed that a $110 \mathrm{kDa}$ precursor form of acid $\alpha$-glucosidase is posttranslationally modified into a $76 \mathrm{kDa}$ mature form and a minor $70 \mathrm{kDa}$ component via a $95 \mathrm{kDa}$ intermediate (Hasilik and Neufeld 1980; Wisselaar et al. 1993; Moreland et al. 2005).

Glycosyl hydrolases have been classified into 77 families based on amino acid sequence similarity, and human acid $\alpha$-glucosidase belongs to family 31 that includes glycosyl hydrolases from mammals to plants (Davies and Henrissat 1995). Although the crystal structure of human acid $\alpha$-glucosidase has not been determined, its catalytic domain is evolutionarily conserved (Lovering et al. 2005; Ernst et al. 2006).

Here we performed biochemical analyses on cultured fibroblasts from patients with Pompe disease and a subject who was thought to have "a pseuodeficiency of acid $\alpha$-glucosidase" as samples, and we constructed structural models of the catalytic domain and the surrounding region of human acid $\alpha$-glucosidase with amino acid substitutions. The aim was to obtain further insight into the molecular pathology of Pompe disease and to determine the difference between the disease and a "pseudodeficiency of acid $\alpha$-glucosidase."

\section{Materials and methods}

Patients and cell culture

Cultured skin fibroblasts were obtained from four patients with Pompe disease (F91, F660, F661 and F664), a subject having low acid $\alpha$-glucosidase activity with no glycogen storage in the organs (F277), and ten controls including F258 with the agreement of the patients and/or their parents to use the cells for this study. The ethical committee of our institute approved the study. The patients' data including their phenotypes and genotypes are summarized in Table 1. All of them were from unrelated Japanese families. Two patients exhibited the infantile-onset phenotype. One of them (F91) was homozygous for p.C103X (Hermans et al. 2004), and the other (F661) had p.R600C on one allele, the mutation on the other allele being unknown (Tsujino et al. 2000). Two patients exhibited the late-onset phenotype. One of them (F664) was homozygous for p.R437C (Kanazawa et al. 2003), and the other (F660) was compound heterozygous for p.S529V and p.S619R (Tsunoda et al. 1996; Kanazawa et al. 2003). One case (F277) was previously reported to have a $K m$ mutant acid $\alpha$-glucosidase for glycogen (Suzuki et al. 1988). This male patient developed cardiac arrhythmia at 12 years of age and died of cardiac failure at 14 years of age. No skeletal muscle involvement was observed either clinically or histologically. No accumulation of glycogen was found in his organs either pathologically or biochemically. Afterward, detailed examination by us revealed that the $K m$ values of acid $\alpha$-glucosidase for both glycogen and an artificial substrate were normal, as described below. Gene analysis revealed that the genotype was p.G576S + p.E689K/ p.G576S + p.E689K (amino acid substitution p.E689K has already been reported to be a neutral polymorphism; Huie et al. 1996; Shieh and Lin 1998), and the genotype of the subject was the same as that found in the asymptomatic cases in Taiwan reported by Hwu et al. So, it was thought that the subject occasionally developed an idiopathic cardiac disorder, but did not have Pompe disease. As far as we 
Table 1 Phenotypes, genotypes and acid $\alpha$-glucosidase activity of the Pompe disease patients and the subject having a "pseudodeficiency of acid $\alpha$-glucosidase"

\begin{tabular}{|c|c|c|c|c|c|}
\hline \multirow[t]{2}{*}{ Case (cultured fibroblasts) } & \multirow[t]{2}{*}{ Phenotype } & \multirow[t]{2}{*}{ Genotype } & \multirow[t]{2}{*}{ Reference } & \multicolumn{2}{|c|}{ Acid $\alpha$-glucosidase activity } \\
\hline & & & & MUG & Glycogen \\
\hline F91 & Infantile-onset & p.C103X/p.C103X & Hermans et al. (2004) & ND & ND \\
\hline F277 & "Pseudodeficiency" & $\begin{array}{l}\text { p.G576S + p.E689K/ } \\
\text { p.G576S + p.E689K }\end{array}$ & Suzuki et al. (1988) & 17 & 0.2 \\
\hline F660 & Late-onset & p.S529V/p.S619R & $\begin{array}{l}\text { Tsunoda et al. (1996) and } \\
\text { Kanazawa et al. (2003) }\end{array}$ & 1 & - \\
\hline F661 & Infantile-onset & p.R600C/undefined & Tsujino et al. (2000) & ND & - \\
\hline F664 & Late-onset & p.R437C/p.R437C & Kanazawa et al. (2003) & 1 & - \\
\hline
\end{tabular}

Acid $\alpha$-glucosidase activity in cultured fibroblasts was measured using MUG and glycogen as substrates, and is expressed as nmol methylumbelliferone released/h/mg protein and $\mu \mathrm{mol}$ glucose released/h/mg protein, respectively

The values for controls were $108 \pm 42[10]$ (mean \pm SD $[n]$ ) for MUG, and 1.9 (F258) for glycogen

Gene analysis of F277 was performed by Dr. Arnold J.J. Reuser

p.E689K has been reported to be a polymorphism (Huie et al. 1996; Shieh and Lin 1998)

$N D$ not detected

know, no subjects exhibiting specific manifestations or signs for Pompe disease have been found among cases homozygous for p.G576S + p.E689K. So, they are thought to have a "pseudodeficiency of acid $\alpha$-glucosidase," although they should be carefully observed.

The cells were cultured in Ham's F-10 medium containing $10 \%$ fetal calf serum and antibiotics at $37^{\circ} \mathrm{C}$ in a humidified incubator flushed continuously with a $5 \% \mathrm{CO}_{2}$ $95 \%$ air mixture.

\section{Assaying of acid $\alpha$-glucosidase}

Acid $\alpha$-glucosidase activity was determined fluorometrically in a total homogenate of cultured fibroblasts using 4-methylumbelliferyl $\alpha$-D-glucopyranoside (MUG) (Sigma, St Louis, MO) as a substrate. Briefly, $10 \mu \mathrm{l}$ of the cell homogenate was added to $40 \mu \mathrm{l}$ of the substrate solution comprising $4 \mathrm{mM}$ MUG in $50 \mathrm{mM}$ citrate buffer, $\mathrm{pH}$ 4.0. The reaction mixture was incubated at $37^{\circ} \mathrm{C}$ for $30 \mathrm{~min}$, and then the reaction was stopped by the addition of $950 \mu \mathrm{l}$ of $0.2 \mathrm{M}$ glycine buffer, $\mathrm{pH} 10.7$. Then, the fluorescence intensity was measured with a spectrofluorometer (F2000; Hitachi, Tokyo, Japan) at excitation and emission wavelengths of 365 and $450 \mathrm{~nm}$, respectively.

Acid $\alpha$-glucosidase activity was also measured with glycogen as a substrate using cells of F91 (infantile-onset Pompe disease), F277 (a "pseudodeficiency of acid $\alpha$-glucosidase"), and F258 (control) as samples. Glycogendegrading activity was measured using mussel glycogen (Sigma-Aldrich, St Louis, MO) as a substrate. Briefly, $12 \mu \mathrm{l}$ of the cell homogenate was added to $48 \mu$ of the substrate solution comprising $62.5 \mathrm{mg} / \mathrm{ml}$ glycogen in $0.1 \mathrm{M}$ citrate$0.2 \mathrm{M}$ sodium phosphate buffer, $\mathrm{pH} 4.0$, followed by incubation for $1 \mathrm{~h}$ at $37^{\circ} \mathrm{C}$. The reaction was terminated by heating at $95^{\circ} \mathrm{C}$ for $2 \mathrm{~min}$. Then, the reaction tube was immediately cooled on ice and centrifuged. An aliquot of the supernatant was subjected to quantitative analysis for liberated glucose with Aspergillus niger glucose oxidase (SigmaAldrich) and horseradish peroxidase (Toyobo, Osaka, Japan) in combination with a sensitive chromogenic compound, 10- $N$-methylcarbanoyl-3,7-bis (dimethylamino) phenothiazine (Kyowa Hedix, Shizuoka, Japan), according to the method described previously (Okumiya et al. 1998). To avoid erroneous results due to turbidity of the glycogen solution and free cellular glucose, the assay was performed without the cell homogenate and without glycogen, and the glycogen-degrading activity was calculated after correcting for the glycogen and sample blanks. As a calibrator for the measurement of glucose, $200 \mu \mathrm{M}$ glucose dissolved in distilled water was used.

Protein determination was performed with a DC assay kit (Bio-Rad, Richmond, CA), using bovine serum albumin as a standard.

Determination of the Michaelis-Menten constants (Kms) of acid $\alpha$-glucosidase for MUG and glycogen

Apparent $K m$ values of acid $\alpha$-glucosidase for MUG and glycogen were determined by means of Lineweaver-Burk plotting using homogenates of fibroblasts from a case with a "pseudodeficiency of acid $\alpha$-glucosidase" (F277) and a control subject (F258).

MUG-degrading activity was measured according to the method described above, the substrate concentration in the assay system being changed (final concentrations: 2, 6, and $10 \mathrm{mM})$.

Glycogen-degrading activity was measured according to the method described above, the substrate concentration in 
the assay system being changed (final concentrations: 3.1, $6.2,12.5,25,50$, and $100 \mathrm{mg} / \mathrm{ml}$ ), and the reaction was terminated after incubation for $4 \mathrm{~h}$ at $37^{\circ} \mathrm{C}$.

\section{Western blot analysis}

Cells (F91, F258, F277, F660, F661, and F664) were sonicated in Milli Q water, and protein concentrations were determined by the method described above. Then, $10 \mu \mathrm{g}$ aliquots of protein in the cell extracts were each solubilized with an equal volume of sodium dodecylsulfate (SDS) sample buffer (62.5 mM Tris-HCl, pH 6.8, 2\% SDS, $10 \%$ glycerol and $5 \% \quad \beta$-mercaptoethanol). Then, they were separated on Tris-glycine polyacrylamide gels (PAG mini; Daiichi Pure Chemical Co. Ltd, Tokyo, Japan), and electrotransferred to polyvinylidene difluoride (PVDF) Immobilon-P membranes (Millipore Co., Bedford, MA). For Western blotting, the blots were blocked in Tris-buffered saline (TBS: $20 \mathrm{mM}$ Tris- $\mathrm{HCl}, \mathrm{pH} 7.5,100 \mathrm{mM}$ $\mathrm{NaCl}$ ) containing $5 \%$ skim milk for 15 min at room temperature. Then, they were reacted with rabbit polyclonal antibodies to human acid $\alpha$-glucosidase (1:500 diluted) (Tsuji and Suzuki 1987), washed in TBS three times, and reacted with peroxidase-conjugated donkey anti-rabbit secondary antibodies (Amersham Pharmacia Biotech, Arlington Heights, IL) for $1 \mathrm{~h}$ at room temperature. Finally, the blots were washed in TBS three times and then developed with ECL (Amersham Pharmacia Biotech) as a chemiluminescent substrate on Hyperfilm ${ }^{\mathrm{TM}}$ ECL (Amersham Pharmacia Biotech). Recombinant human acid $\alpha$ glucosidase components $(110,95$ and $76 \mathrm{kDa})$ produced in Chinese hamster ovary cells were used as standards.

Densitometry to determine the amounts of the enzyme components was performed with a GT-X750 scanner (Epson, Tokyo, Japan) and Quantity One software version 4.2 (Bio-Rad Laboratories, Hemel Hempstead, UK).

Structural modeling of the catalytic domain and the surrounding region of human wild-type acid $\alpha$-glucosidase and the enzyme with amino acid substitutions

Structural modeling of the catalytic domain and the surrounding region of wild-type acid $\alpha$-glucosidase and the enzyme with amino acid substitutions, including p.R437C, p.S529R, p.G576S, p.R600C, p.S619R and p.E689K, was performed using molecular modeling software, SYBYL/ BIOPOLYMER (TRIPOS, St Louis, MO). As a template, $\alpha$-glucosidase MalA from Sulfolobus solfataricus (SwissProt Entry Name: AGLU_SULSO, Accession number: O59645, PDB ID: 2G3N), which belongs to glycoside hydrolase family 31 (GH31) (Ernst et al. 2006) was used. According to sequence alignment, a model of the human wild-type acid $\alpha$-glucosidase composed of 452 amino acids (residues 344-795) was modeled based on the template MalA (residues 152-604). The amino acid identity of this region between human acid $\alpha$-glucosidase and MalA was $30.0 \%$. Because the amino acid identity of the region corresponding to the residues 1-151 and 605-693 in MalA are very low, 19.7 and $11.5 \%$, respectively, these regions were not modeled. The energy minimization procedure was performed to optimize the conformations and side-chain rotamers. To determine the influence of an amino acid replacement on the structure, each mutant model was superimposed on the wild-type model based on the $\mathrm{C} \alpha$ atoms by means of the least-square-mean fitting method (Kabsch 1976, 1978). To compare the influence of the amino acid substitutions on the three-dimensional structure between each amino acid substitution and the wild-type model, the root-mean-square distance (rmsd) value for all atoms within $8 \AA$, which is the distance used for the calculation of local conformational changes, from the $\mathrm{C} \alpha$ atom of a substituted amino acid residue was calculated as a local rmsd value (Weiner et al. 1984; Tripos Bookshelf 7.0, Tripos Inc., St Louis, MO).

\section{Results}

Acid $\alpha$-glucosidase activity in cultured fibroblasts

The results of acid $\alpha$-glucosidase assaying are shown in Table 1. In cases F91 (genotype: p.C103X/p.C103X) and F661 (p.R600C/undefined) with infantile-onset Pompe disease, acid $\alpha$-glucosidase activity was absent. Among the cases of late-onset Pompe disease, very low but residual enzyme activity was detected in F660 (p.S529V/p.S619R) and F664 (p.R437C/p.R437C), when sensitive assaying with MUG was performed. In F277, considerable residual activity toward both MUG and glycogen (15 and $11 \%$ of the normal levels, respectively) was detected.

Apparent $K m$ values of acid $\alpha$-glucosidase for MUG and glycogen

The apparent $K m$ values of acid $\alpha$-glucosidase for MUG and glycogen in cultured fibroblasts were measured. The apparent $\mathrm{Km}$ values for MUG in F277 and F258 were $1.8 \pm 0.5[4]$ and $1.6 \pm 0.1[4] \mathrm{mM}$ (mean $\pm \mathrm{SD}[n]$ ), respectively, and those for glycogen in F277 and F258 were 33 and $36 \mathrm{mg} / \mathrm{ml}$ (mean), respectively. The duplicates did not differ by more than $5 \%$. The average values were subjected to linear regression analysis, and the $K m$ values were determined by means of least-square approach. In 
both cases, no difference was found between F277 and F258 in the apparent $K m$ value for either MUG or glycogen. Enzyme assays to determine the $\mathrm{Km}$ values for MUG and glycogen were performed with different concentrations of substrate in quadruplicate and duplicate, respectively.

Acid $\alpha$-glucosidase protein in cultured fibroblasts

The results of Western blotting for acid $\alpha$-glucosidase in cultured fibroblasts are shown in Fig. 1. Bands corresponding to the $95 \mathrm{kDa}$ intermediate and the $76 \mathrm{kDa}$ major mature enzyme protein were apparently detected for a sample from a control subject (F258). The $110 \mathrm{kDa}$ precursor and $70 \mathrm{kDa}$ minor mature components could not be detected. Pompe mutants (F91, F660, F661 and F664) did not give any bands corresponding to acid $\alpha$-glucosidase components on Western blotting followed by densitometry under the experimental conditions used here, and we could not find any difference between the infantile-onset group and the late-onset one. On the other hand, the $95 \mathrm{kDa}$ and $76 \mathrm{kDa}$ enzyme components were observed for F277, the subject having low acid $\alpha$-glucosidase activity, and no glycogen storage was observed in his organs. The amount of the enzyme protein estimated from the results of Western blotting almost corresponded to the residual enzyme activity.

Structural models of the catalytic domain and the surrounding region of human wild-type acid $\alpha$-glucosidase and the enzyme with amino acid substitutions

To investigate the enzyme defect in Pompe disease from a structural perspective, we constructed three-dimensional

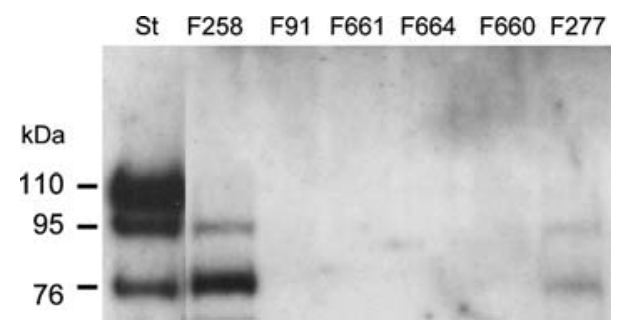

Fig. 1 Western blotting for acid $\alpha$-glucosidase in cultured fibroblasts from patients with Pompe disease, a subject having a "pseudodeficiency of acid $\alpha$-glucosidase," and a control subject. St (recombinant human acid $\alpha$-glucosidase components), F258 (phenotype and genotype: control, wild-type/wild-type), F91 (infantile-onset type, p.C103X/p.C103X), F661 (infantile-onset type, p.R600C/undefined), F664 (late-onset type, p.R437C/p.R437C), F660 (late-onset type, p.S529V/p.S619R), and F277 ("pseudodeficiency", p.G576S + p.E689K/ p.G576S + p.E689K) models of the catalytic domain and the surrounding region (residues 344-795) of human wild-type acid $\alpha$-glucosidase and the enzyme with amino acid substitutions by means of homology modeling. We used here the crystallographic data for family 31 glycosyl hydrolase MalA from Sulfolobus solfataricus (residues 152-604) as a template. First, according to sequence alignment, we built a model of the human wild-type acid $\alpha$-glucosidase composed of 452 amino acids. Figure 2 shows the sequence alignment between human acid $\alpha$-glucosidase (residues $344-795$ ) and the template MalA (residues 152-604). The residues responsible for the enzymatic activity and seven sequence motifs of family 31 glycosyl hydrolases are well conserved (Fig. 2).

The overall superimposed structure of the template MalA (PDB ID: 2G3N chain-A) and the modeled structure of the catalytic domain and the surrounding region of human wild-type acid $\alpha$-glucosidase is shown in Fig. 3. It is folded into a $(\beta / \alpha)_{8}$-barrel with an active site pocket at the $\mathrm{C}$-terminus of each $\beta$-strand. The structure of the active site pocket of human wild-type acid $\alpha$-glucosidase is similar to that of MalA. Catalytic nucleophile D320 and acid/ base D416 found in MalA are identical to D518 and D616 in human acid $\alpha$-glucosidase. Residues D212, R400, D416 and H480, which were deduced to be substrate binding residues in MalA (Ernst et al. 2006), are identical to residues D404, R600, D616 and H674 in human acid $\alpha$-glucosidase, respectively.

There are three potential sites for $N$-linked glycosylation $(\mathrm{N}-\mathrm{X}-\mathrm{S} / \mathrm{T})$ in the catalytic domain. In our model, all the $\mathrm{N}$ residues, i.e., N390 (glycosylation site: $\mathrm{N}-\mathrm{M}-\mathrm{T}$ ), N470 (N-E-T), and N652 (N-T-S), are located on the surface of the molecule, and their side chains are exposed (Fig. 3).

According to the constructed model, there is a disulfide bond (C647-C658) in the catalytic domain structure. C647 and $\mathrm{C} 658$ are located in the loop following $\mathrm{A} \beta$-strand and in A $\alpha$-helix, respectively. N652, which is one of the potential $\mathrm{N}$-linked glycosylation sites, is located in this region stabilized by a disulfide bond between C647 and C658. So, this disulfide bond is thought to be very important for stabilization of the molecule.

The positions of residues where amino acid substitutions have been detected were mapped in the modeled threedimensional structure of the human wild-type acid $\alpha$-glucosidase (Fig. 4). To determine the influence of the amino acid substitutions, we constructed amino acid-substituted models and compared them with the wild-type structure.

The R600 residue is located between the $\mathrm{A} \beta 5$ and $\mathrm{A} \beta 5^{\prime}$ strands (Fig. 2) and corresponds to R400 involved in substrate binding in MalA (Ernst et al. 2006). Substitution of R600 with $\mathrm{C}$ affects the main chain of the barrel structure as well as active site residues D518 and D616 (Fig. 5a). The local rmsd between the wild-type and p.R600C was $0.23 \AA$ (Table 2). 


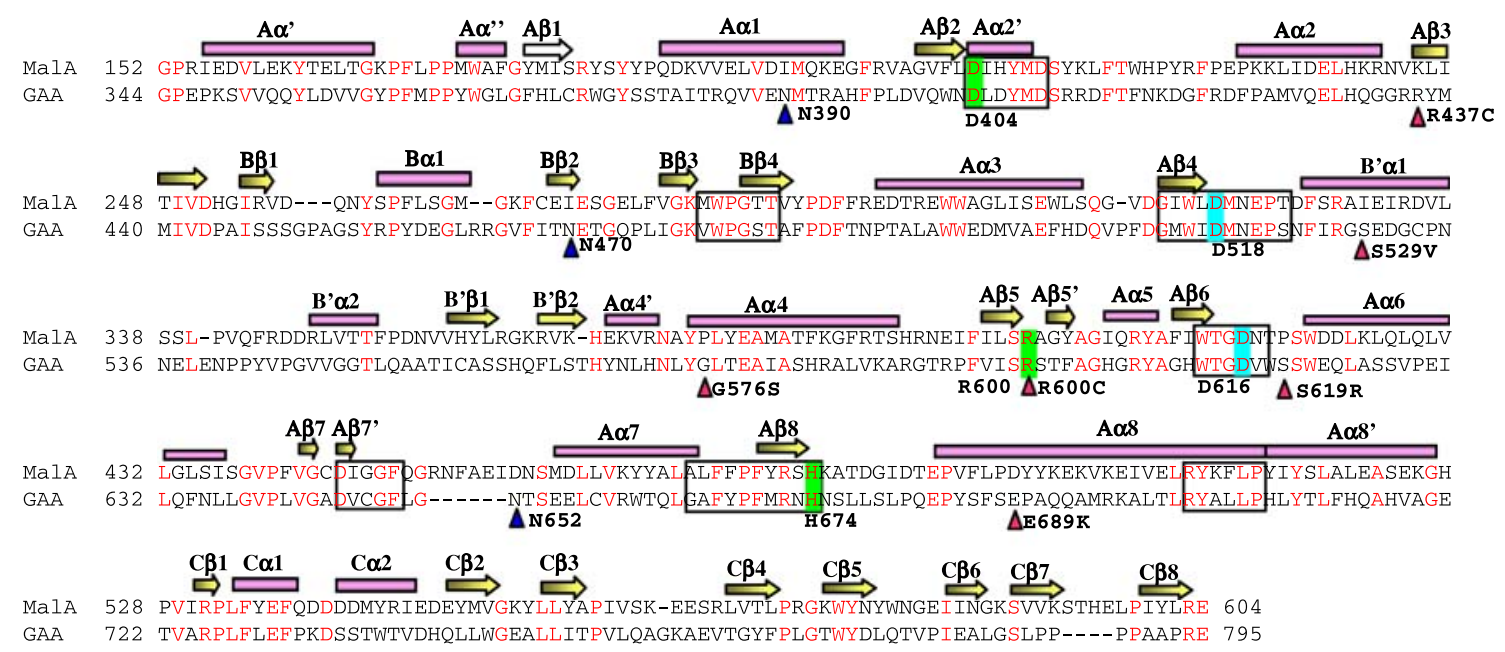

Fig. 2 Sequence alignment of human acid $\alpha$-glucosidase (residues 344-795) and the template MalA (residues 152-604). Catalytic residues and substrate binding residues are colored cyan and green, respectively. $\mathrm{N}$-Glycosylation sites and mutation sites are indicated by

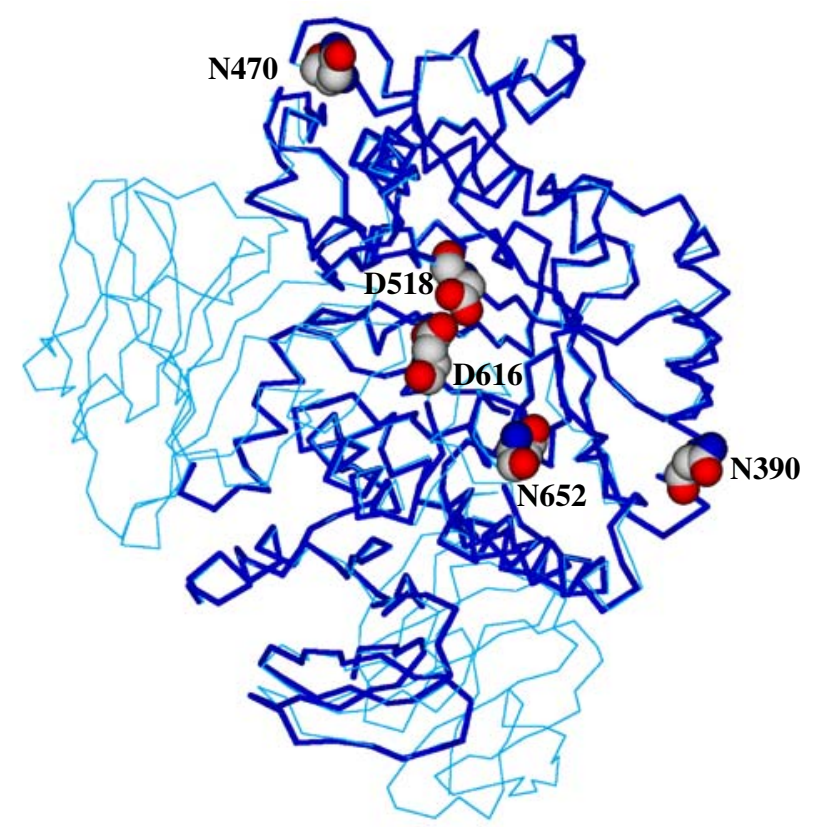

Fig. 3 Superimposed $\mathrm{C} \alpha$ traces of the human wild-type acid $\alpha$-glucosidase model and the template MalA. The acid $\alpha$-glucosidase and MalA are shown in blue and cyan, respectively. Catalytic nucleophile D518, acid/base D616, and N residues $(N 390, N 470$ and N652) at potential glycosylation sites are shown as a space-filling model

The S619 residue is located in the loop following $\mathrm{A} \alpha$ helix (Fig. 2), and its side chain is packed into loop structures. Substitution of S619 with a larger amino acid residue, $R$, is predicted to cause structural changes of the main chains in the two loops comprising G651-E656 and L678-P681. The local rmsd between the wild-type and blue triangles and red triangles, respectively. Conserved sequence regions of GH31 are surrounded by boxes. Secondary structure schemes of MalA are shown with reference to those by Ernst et al. (2006)

p.S619R was $0.34 \AA$ (Table 2), and the maximum influenced distance was $1.59 \AA$. This substitution is deduced to cause the largest structural change among the six mutations.

The R437 residue is located at the N-terminal end of $\mathrm{A} \beta$-strand (Fig. 2). The side chain of R437 is exposed on the surface of the opposite side of the active site pocket (Fig. 4). Substitution of R437 with C causes structural changes of mainly the side chains on the surface (Fig. 5c). The local rmsd between the wild-type and p.R437C was $0.21 \AA$ (Table 2).

The G576 residue is located in A $\alpha 4$-helix (Fig. 2) and inside the barrel structure. Substitution G576 with S causes conformational changes of the side chains on the helix (Fig. 5d). This structural change is small, and the local rmsd between the wild-type and p.G576S was $0.18 \AA$ (Table 2). This limited conformational change caused by p.G576S is thought not to affect active site D518 or binding site R600.

The E689 residue is located at the $N$-terminus of $\mathrm{A} \alpha 8$ helix (Fig. 2), and its side chain is fully exposed (Fig. 4). Amino acid substitution of E689 with $\mathrm{K}$ little affected the three-dimensional structure of acid $\alpha$-glucosidase (Fig. 5e), and the local rmsd was $0.06 \AA$ (Table 2). p.G576S and p.E689K do not influence each other because there is 45.4 $\AA$ between the $\mathrm{C}_{\alpha}$ atoms of G576 and E689.

The $\mathrm{S} 529$ residue is located in $\mathrm{B}^{\prime} \alpha 1$ of the subdomain $\mathrm{B}^{\prime}$ (Fig. 4). As this region is packed, another subunit in MalA, the subdomain $\mathrm{B}^{\prime}$ also might be interacted with other region of human acid $\alpha$-glucosidase. However, as the model constructed here was limited, the region interacted spatially with S529 of subdomain B' could not be modeled (Fig. 4). So, we could not determine the structural influence of p.S529V based on rmsd in this case. 
Fig. 4 Positions of Pompe mutations mapped on the structure of human wild-type acid $\alpha$-glucosidase. The structure of the catalytic domain is shown as a ribbon model. The residues involved in the amino acid substitutions responsible for Pompe disease (R437, S529, $R 600$ and $S 619)$, a "pseudodeficiency of acid $\alpha$-glucosidase" (G576), and a neutral polymorphism (E689) are shown as a space-filling model
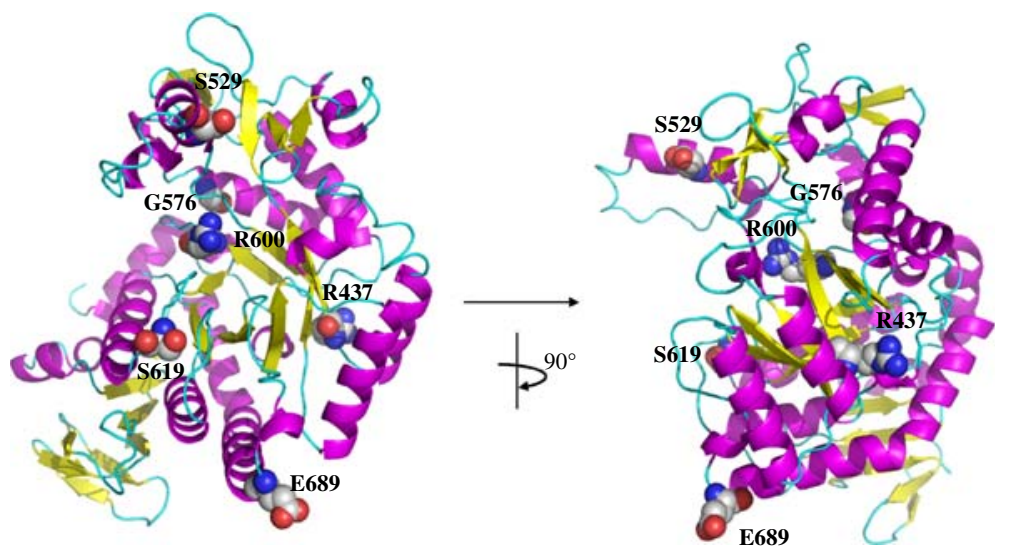

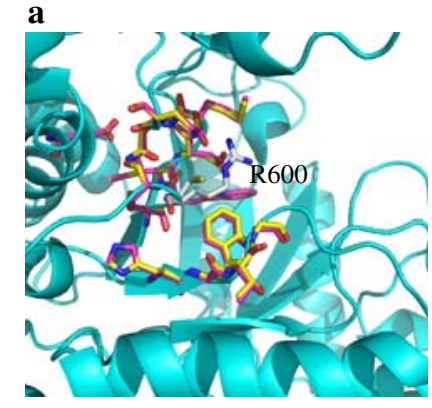

b
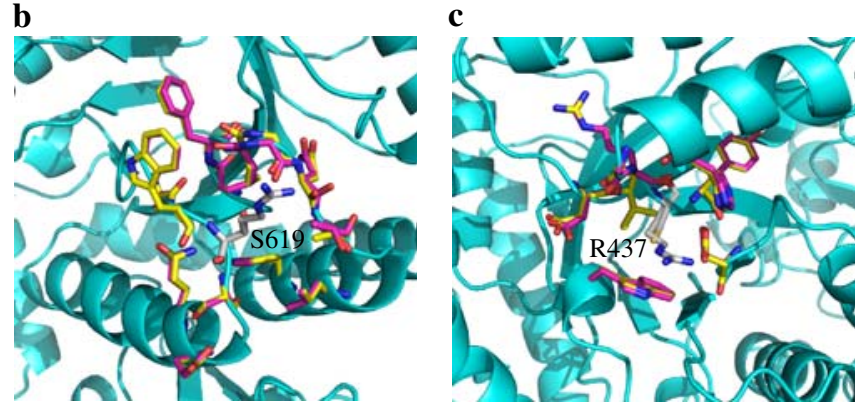

e d

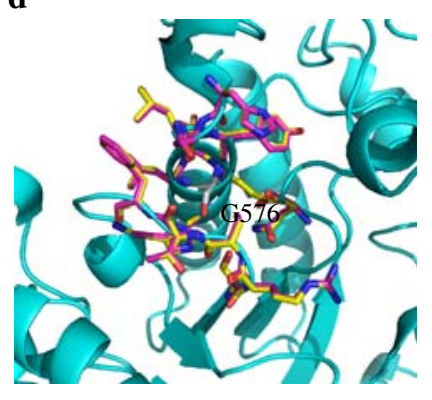

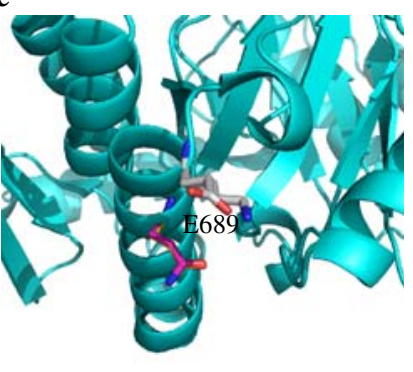

Fig. 5 Predicted structural changes in human acid $\alpha$-glucosidase caused by amino acid substitutions. p.R600C (a), p.S619R (b), p.R437C (c), p.G576S (d), and p.E689R (e). The whole structure is shown as a ribbon model colored cyan. We defined the structure as

Table 2 Local root mean square distances for all atoms within $8 \AA$ of the $\alpha$ carbon of substituted amino acid residue in the mutant models

\begin{tabular}{ll}
\hline Amino acid substitution & Local rmsd $(\AA)$ \\
\hline R437C & 0.21 \\
G576S & 0.18 \\
R600C & 0.23 \\
S619R & 0.34 \\
E689K & 0.06 \\
\hline
\end{tabular}

\section{Discussion}

Pompe disease exhibits clinical and biochemical heterogeneity (Hirschhorn and Reuser 2001; Hermans et al. 2004; being influenced by an amino acid replacement when the position of an atom of the mutant differed from that of the wild-type by more than the average total rmsd value $(0.10 \AA)$. The influenced residues of the wild-type (magenta) and mutants (yellow) are shown as sticks

Reuser et al. 1985, 1987). The details of the molecular pathology of Pompe disease have not been clarified yet, although gene analyses have revealed many gene mutations responsible for this disease. As efficient enzyme replacement therapy for Pompe disease involving recombinant human acid $\alpha$-glucosidases has been reported (Van den Hout et al. 2004; Klinge et al. 2005; Kishnani et al. 2006), and it has been recognized that there are people whose acid $\alpha$-glucosidase activity is low but who do not exhibit any clinical manifestations of Pompe disease, new knowledge for further understanding of Pompe disease is desired to facilitate therapeutic planning.

In this study, we performed structural and biochemical analyses to obtain further insight into the molecular basis 
of this disease and to determine the difference between the disease and a "pseudodeficiency of acid $\alpha$-glucosidase."

F91 was homozygous for p.C103X. As such a nonsense mutation at the N-terminal end of a protein should cause loss of the active site and thus result in destabilization of the enzyme protein and/or a decrease in the amount of acid $\alpha$-glucosidase mRNA, enzyme activity was absent.

F661 had a missense mutation, p.R600C, the second mutation being as yet undefined. As a different Pompe disease patient who was homozygous for p.R600C had the infantile-onset form and did not exhibit any acid $\alpha$-glucosidase activity (Tsujino et al. 2000), p.R600C is thought to seriously affect acid $\alpha$-glucosidase. Considering the results of enzyme assaying and Western blotting, the expressed mutant enzyme protein with p.R600C is thought to be unstable and to undergo degradation. Structural analysis revealed that p.R600C affects the barrel structure as well as the active site, and this finding is not inconsistent with that on biochemical analysis that acid $\alpha$-glucosidase activity was absent. As no bands corresponding to the enzyme protein were detected for F661 on Western blotting, the second mutation would not allow the production of any protein or only allow the expression of a very unstable one.

F660 was genotypically compound heterozygous for p.S529V and p.S619R. Our structural study revealed that p.S619R causes a large structural change around residue S619, which results in dislocation of N652, which is one of the potential $N$-glycosylation sites. This structural change is thought to affect the stability of the protein and to result in a deficiency of acid $\alpha$-glucosidase activity.

S529 was deduced to be located in $\mathrm{B}^{\prime} \alpha 1$ of the subdomain $\mathrm{B}^{\prime}$, which is packed another subunit in MalA. Unfortunately, the region closing around S529 spatially was not modeled here, and thus we could not discuss the local rmsd value between the wild type and p.S529V. A previous report suggested that p.S529V leads to late-onset Pompe disease (Tsunoda et al. 1996). The subdomain $\mathrm{B}^{\prime}$, which S529 is located on, is conserved among the majority of GH31 enzymes and is adjacent to the catalytic pocket. Then, the subdomain $\mathrm{B}^{\prime}$ may have a critical role in substrate binding (Ernst et al.2006). Considering the results of Western blotting, the amount of the mutant enzyme protein was apparently decreased, and p.S529V may also cause destabilization of the molecule.

F664 is genotypically homozygous for p.R437C. Our structural analysis revealed that p.R437C causes a structural change on the surface of the molecule. This would allow very low residual enzyme activity.

F277 was genotypically homozygous for p.G576S + p.E689K. Structural analysis revealed that p.E689K, which is known to be a neutral polymorphism, hardly affects the three-dimensional structure of acid $\alpha$-glucosidase and does not affect p.G576S structurally. As p.G576S causes a small conformational change of side chains and does not affect the active site of the enzyme, it is thought that the expressed protein is unstable, but exhibits considerable residual activity and normal affinity for substrates. This considerable residual enzyme activity probably prevents the accumulation of glycogen.

Thus, mutations causing apparent structural changes in acid $\alpha$-glucosidase are thought to lead to a decrease in the enzyme activity, resulting in Pompe disease. We could not detect any difference between the infantile-onset group and the late-onset one on Western blotting followed by densitometry. As preliminary studies revealed that the antibodies against acid $\alpha$-glucosidase used here could not detect $<2.5$ nanograms of the antigen (data not shown), it is thought that the very small amounts of residual enzyme protein in F660 and F664 could not be detected on Western blotting under the experimental conditions used. On the other hand, mutation p.G576S responsible for a "pseudodeficiency of acid $\alpha$-glucosidase" causes a small conformational change that results in considerable levels of enzyme protein and residual activity.

In conclusion, we constructed a structural model of the catalytic domain and the surrounding region of human acid $\alpha$-glucosidase, and investigated the structural consequences of amino acid substitutions in acid $\alpha$-glucosidase to increase our molecular understanding of Pompe disease.

Acknowledgments We thank Dr. W.L. Hwu (Department of Pediatrics and Medical Genetics, National Taiwan University Hospital, Taipei, Taiwan) and Dr. A.J.J. Reuser (Department of Clinical Genetics, Ersmus MC, Rotterdam, The Netherlands) for the valuable discussions. This work was partly supported by grants from the Japan Society for the Promotion of Science, the Ministry of Education, Science, Sports and Culture of Japan, the Ministry of Health, Labor and Welfare of Japan, the Japan Science and Technology Agency, and CREST.

\section{References}

Davies G, Henrissat B (1995) Structures and mechanisms of glycosyl hydrolases. Structure 3:853-859

Ernst HA, Leggio LL, Willemoës M, Leonard G, Blum P, Larsen S (2006) Structure of the Sulfolobus solfataricus $\alpha$-glucosidase: implications for domain conservation and substrate recognition in GH31. J Mol Biol 358:1106-1124

Hasilik A, Neufeld EF (1980) Biosynthesis of lysosomal enzymes in fibroblasts. Phosphorylation of mannose residues. J Biol Chem 255:4946-4950

Hermans MM, van Leenen D, Kroos MA, Beesley CE, Van Der Ploeg AT, Sakuraba H, Wevers R, Kleijer W, Michelakakis H, Kirk EP, Fletcher J, Bosshard N, Basel-Vanagaite L, Besley G, Reuser AJ (2004) Twenty-two novel mutations in the lysosomal alpha-glucosidase gene (GAA) underscore the genotype-phenotype correlation in glycogen storage disease type II. Hum Mutat 23:47-56

Hirschhorn R, Reuser AJJ (2001) Glycogen storage disease type II: acid alpha-glucosidase (acid maltase) deficiency. In: Scriver CR, 
Beaudet AL, Sly WS, Valle D (eds) The metabolic and molecular bases of inherited disease. 8th edn. McGraw-Hill, New York, pp 3389-3420

Hoefsloot LH, Hoogeveen-Westerveld M, Kroos MA, van Beeumen J, Reuser AJ, Oostra BA (1988) Primary structure and processing of lysosomal alpha-glucosidase; homology with the intestinal sucrase-isomaltase complex. EMBO J 7:1697-1704

Hoefsloot LH, Hoogeveen-Westerveld M, Reuser AJ, Oostra BA (1990) Characterization of the human lysosomal alpha-glucosidase gene. Biochem J 272:493-497

Huie ML, Menaker M, McAlpine PJ, Hirschhorn R (1996) Identification of an E689K substitution as the molecular basis of the human acid alpha-glucosidase type 4 allozyme (GAA*4). Ann Hum Genet 60:365-368

Kabsch W (1976) A solution for the best rotation to relate two sets of vectors. Acta Cryst A32:922-923

Kabsch W (1978) A discussion of the solution for the best rotation to relate two sets of vectors. Acta Cryst A34:827-828

Kanazawa N, Miyamoto T, Ihara K, Miyoshi Y, Sakai N, Inui K, Hagiwara K, Kawamoto M, Kouhara N, Sasaki M, Sugie H, Sakai F, Tsujino S (2003) Novel mutation and a frequent mutation in Japanese juvenile patients with acid maltase deficiency. J Inher Metab Dis 26(Supple 2):310

Kishnani PS, Nicolino M, Voit T, Rogers RC, Tsai AC, Waterson J, Herman GE, Amalfitano A, Thurberg BL, Richards S, Davison M, Corzo D, Chen YT (2006) Chinese hamster ovary cellderived recombinant human acid alpha-glucosidase in infantileonset Pompe disease. J Pediatr 149:89-97

Klinge L, Straub V, Neudorf U, Schaper J, Bosbach T, Gorlinger K, Wallot M, Richards S, Voit T (2005) Safety and efficacy of recombinant acid alpha-glucosidase (rhGAA) in patients with classical infantile Pompe disease: results of a phase II clinical trial. Neuromuscul Disord 15:24-31

Lovering AL, Lee SS, Kim YW, Withers SG, Strynadka NC (2005) Mechanistic and structural analysis of a family 31 alphaglycosidase and its glycosyl-enzyme intermediate. J Biol Chem 280:2105-2115

Martiniuk F, Bodkin M, Tzall S, Hirschhorn R (1991) Isolation and partial characterization of the structural gene for human acid alpha glucosidase. DNA Cell Biol 10:283-292

Martiniuk F, Mehler M, Pellicer A, Tzall S, La Badie G, Hobart C, Ellenbogen A, Hirschhorn R (1986) Isolation of a cDNA for human acid alpha-glucosidase and detection of genetic heterogeneity for mRNA in three alpha-glucosidase-deficient patients. Proc Natl Acad Sci USA 83:9641-9644

Moreland RJ, Jin X, Zhang XK, Decker RW, Albee KL, Lee KL, Cauthron RD, Brewer K, Edmunds T, Canfield WM (2005)
Lysosomal acid alpha-glucosidase consists of four different peptides processed from a single chain precursor. J Biol Chem 280:6780-6791

Okumiya T, Jiao Y, Saibara T, Miike A, Park K, Kageoka T, Sasaki M (1998) Sensitive enzymatic assay for erythrocyte creatine with production of methylene blue. Clin Chem 44:1489-1496

Reuser AJ, Kroos M, Oude Elferink RP, Tager JM (1985) Defects in synthesis, phosphorylation, and maturation of acid alpha-glucosidase in glycogenosis type II. J Biol Chem 260:8336-8341

Reuser AJ, Kroos M, Willemsen R, Swallow D, Tager JM, Galjaard H (1987) Clinical diversity in glycogenosis type II. Biosynthesis and in situ localization of acid alpha-glucosidase in mutant fibroblasts. J Clin Invest 79:1689-1699

Shieh JJ, Lin CY (1998) Frequent mutation in Chinese patients with infantile type of GSD II in Taiwan: evidence for a founder effect. Hum Mutat 11:306-312

Suzuki Y, Tsuji A, Omura K, Nakamura G, Awa S, Kroos M, Reuser AJ (1988) Km mutant of acid alpha-glucosidase in a case of cardiomyopathy without signs of skeletal muscle involvement. Clin Genet 33:376-385

Tsuji A, Suzuki Y (1987) The precursor of acid alpha-glucosidase is synthesized as a membrane-bound enzyme. Biochem Int 15:945952

Tsujino S, Huie M, Kanazawa N, Sugie H, Goto Y, Kawai M, Nonaka I, Hirschhorn R, Sakuragawa N (2000) Frequent mutations in Japanese patients with acid maltase deficiency. Neuromuscul Disord 10:599-603

Tsunoda H, Ohshima T, Tohyama J, Sasaki M, Sakuragawa N, Martiniuk F (1996) Acid alpha-glucosidase deficiency: identification and expression of a missense mutation (S529V) in a Japanese adult phenotype. Hum Genet 97:496-499

Van den Hout JM, Kamphoven JH, Winkel LP, Arts WF, De Klerk JB, Loonen MC, Vulto AG, Cromme-Dijkhuis A, WeisglasKuperus N, Hop W, Van Hirtum H, Van Diggelen OP, Boer M, Kroos MA, Van Doorn PA, Van der Voort E, Sibbles B, Van Corven EJ, Brakenhoff JP, Van Hove J, Smeitink JA, de Jong G, Reuser AJ, Van der Ploeg AT (2004) Long-term intravenous treatment of Pompe disease with recombinant human alphaglucosidase from milk. Pediatrics 113:e448-457

Weiner SJ, Kallman PA, Case DA, Singh UC, Ghio C, Alagona G, Profeta S, Weiner P (1984) A new force field for molecular mechanical simulation of nucleic acids and proteins. J Am Chem Soc 106:765-784

Wisselaar HA, Kroos MA, Hermans MM, van Beeumen J, Reuser AJ (1993) Structural and functional changes of lysosomal acid alpha-glucosidase during intracellular transport and maturation. J Biol Chem 268:2223-2231 